\title{
Study on the Effect of Zizyphus Jujuba 'Zhanshanmizao' Dormancy Release by Cover
}

\author{
Lei Li ${ }^{1, a}$, Qunxian Deng ${ }^{1, b *}$, Yan Zhou ${ }^{1, c}$, Xuehui Lan ${ }^{1, d}$ \\ ${ }^{1}$ College of Horticulture, Sichuan Agricultural University, Chengdu, Sichuan, China \\ a736984004@qq.com, b1324856299@qq.com, 'czhouyan6213500@qq.com, d563590085@qq.com \\ ${ }^{*}$ Corresponding author
}

Keywords: Ziziphus jujuba 'Zhanshanmizao'; shed; dormancy; water; nutrients

Abstract. In order to explore the effect of covering on the natural dormancy of jujube, 7-year-old Ziziphus jujuba 'Zhanshanmizao' was used to study physiological index such as the content of water, Carbohydrate, soluble protein and free amino acid by different shed time (December 5 th, 2016, December 25 th, 2016, January 16 th, 2017). The results showed that: Compared with open field cultivation, there were differences in physiological index content and variation trend of jujube trees after covering. The total water content and free water content of jujube branches treated with shed cultivation were higher than open field cultivation, while the bound water content was lower. Compared with open field cultivation, covering makes the dormancy release early. In the natural dormancy of jujube tree (December 6 th to December 20 th), the total water content, free water content, and soluble protein content decreased, while the bound water content, starch, soluble sugar content, and free amino acid content of the branches increased.

\section{Introduction}

Due to the chilling requirement, the majority of fruit trees such as peach, apricot, pear, and grapes to promote fruit maturity early to March, but still cannot be listed before the Spring Festival, thus restricting the annual supply of fruits [1]. At present, there are two main methods to promote the ripening of fruits early by controlling dormancy artificially. One is to let fruit trees do not enter true dormancy and allow them to continue growing (without dormant), Xia make jujube tree do not enter the true dormancy to achieve the annual ripening by shed and chemical treatment [2]; the second is to make trees leave the deep dormancy as soon as possible and do not be delayed by the ecological dormancy. Medicament treatment could make grape mature early in the dormant period [3]. The dormancy of deciduous fruit trees is a complex physiological process [4,5]. In recent years, researchers have gradually paid more attention to the dormancy mechanism and material metabolism of bud and branches [6,7]. In this experiment, we studied the physiological changes of jujube trees during the natural dormancy and the effect of breaking dormancy by different shed time; our purpose was to provide theoretical basis for promote germination and flowering early in the year-round production.

\section{Materials and methods}

Samples and Treatments. Orchard is located in Yonglian Village, Santai County, Sichuan Province, China $\left(31^{\circ} 16^{\prime} \mathrm{N}, 105^{\circ} 24^{\prime} \mathrm{E}\right)$. The test material was 7 a jujube (tillering propagation seedling) at a planting space of $2 \mathrm{~m} \times 3 \mathrm{~m}$. Covered on December 5 th, 2016 (T1 treatment), December 25 th, 2016 (T2 treatment), January16 th, 2017 (T3 treatment), with open field cultivation as control (CK). 6 plants as 1 plot, each treatment was repeated 4 times.

The first sampling was performed on the second day after covering, from December 5 th, 2016 to March 13 th, 2017, 3-5 representative branches of jujube were collected from the middle and upper part of the crown of jujube tree every 14 days, branches were wrapped in plastic wraps and placed in ice box, then brought back to lap. 
Methods. The content of total water, free water and bound water were determined by Wang's method [8], Starch and soluble sugar content were determined by anthrone colorimetry [8], coomassie brilliant blue g-250 method was used to analyze soluble protein content [8], the content of free amino acids was determined based on ninhydrin colorimetry [8].

Statistical Analyses. Using Excel 2010 and SPSS 20.0 for data analysis.

\section{Results and Discussion}

The Total Water Content. The content of total water in the branches of Ziziphus jujuba 'Zhanshanmizao' decreased firstly and then increased (Fig. 1), shed cultivation was higher than open field cultivation and T3 was higher than all other treatments. The change trend is consistent with the research result of Gao [9]. The time of covering shelter did not change the trend of the total water content, but the effect was different. All 4 treatments reached minimum value on December 20 th, as jujube tree had entered the deepest dormancy. Compared with December 20 th, the total water content of T1, T2, T3, and CK increased 10.94\%, 16.56\%, 17.88\%, and 12.89\% respectively, on March 13 th. The increased degree of T3 was greatest and the total water content was highest, which was most active before germination. Among the shed treatment, the effect of T3 was best.

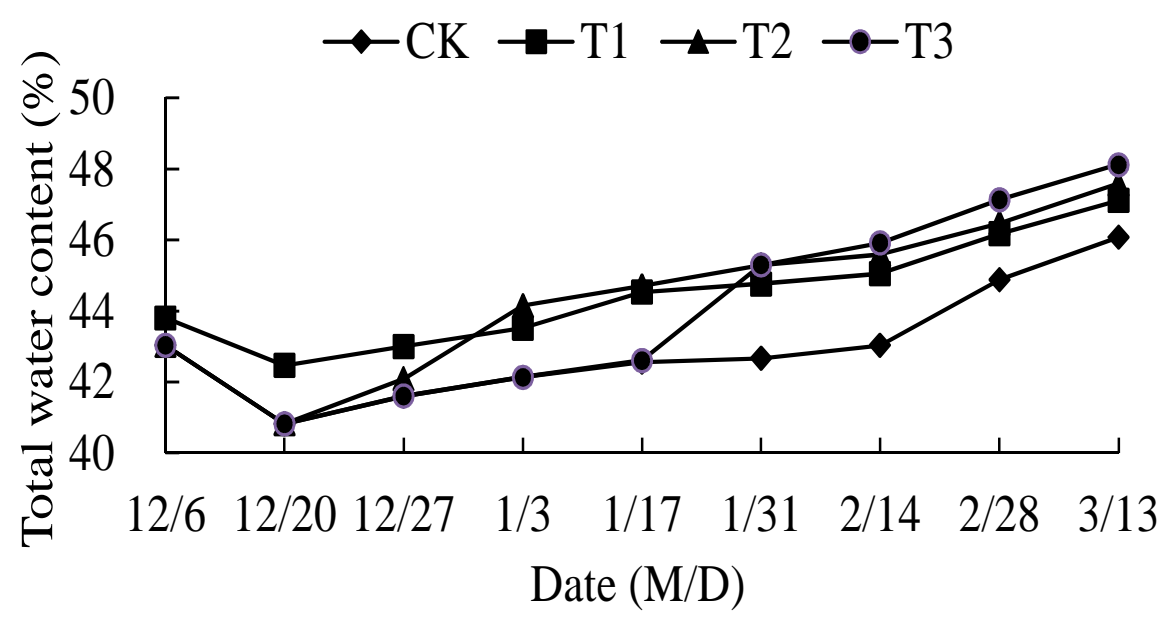

Fig. 1 Change of total water content

The Free Water Content. From December 6 th to March 13 th, the content of free water in the branches decreased firstly and then increased which has same trend of the total water (Fig. 2), but the variation was bigger. Compared with December 20 th, the total water content of T1, T2, T3, and CK increased $145.71 \%, 187.65 \%, 192.32 \%$, and 135.72\% respectively, on March 13 th. The increased degree of T3 $>$ T2 $>$ T1. In the deep dormancy period, the content of total water and free water in the branches of jujube tree were decreased so that jujube tree could increase the resistance to cold and avoid the cell space icing.

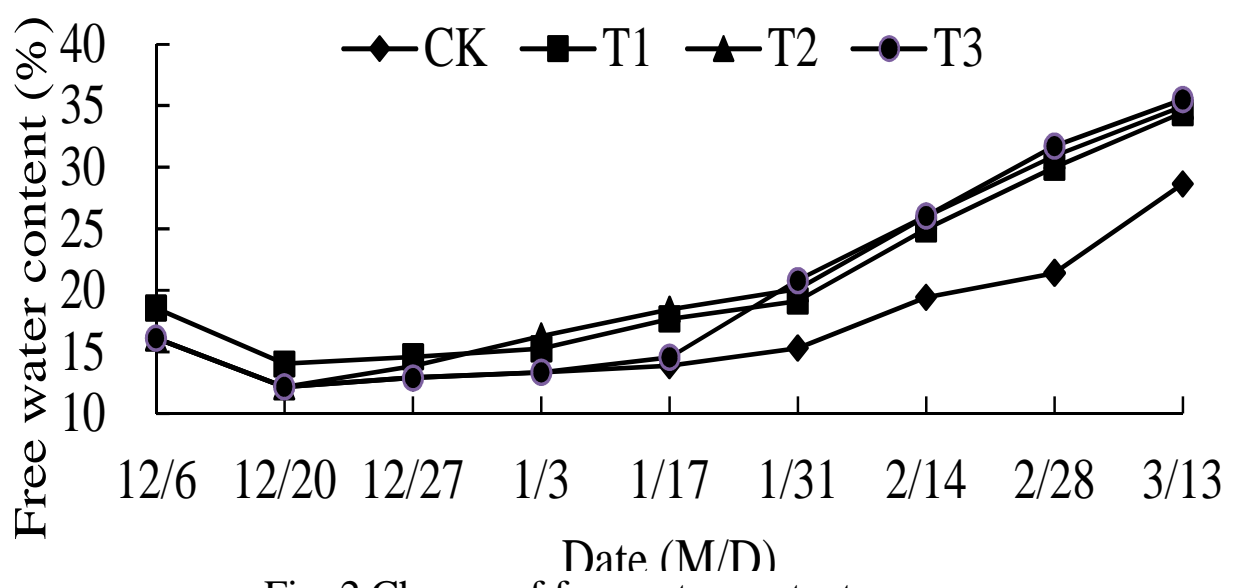

Fig. 2 Change of free water content 
The Bound Water Content. From December 6 th to March 13 th, the bound water content increased firstly and then decreased (Fig. 3), which was contrary to the trend of the total water and free water. For the bound water content, open filed cultivation was higher than shed cultivation beacause the jujube tree had to enhance the cold resistance of in low temperature outside. Compared with December 20th, the bound water content of T1, T2, T3, and CK increased 55.54\%, 55.95\%, 56.05\%, and $39.17 \%$ respectively, on March 13 th. The decrease amplitude of T3 was greatest, so the effect of T3 was best.

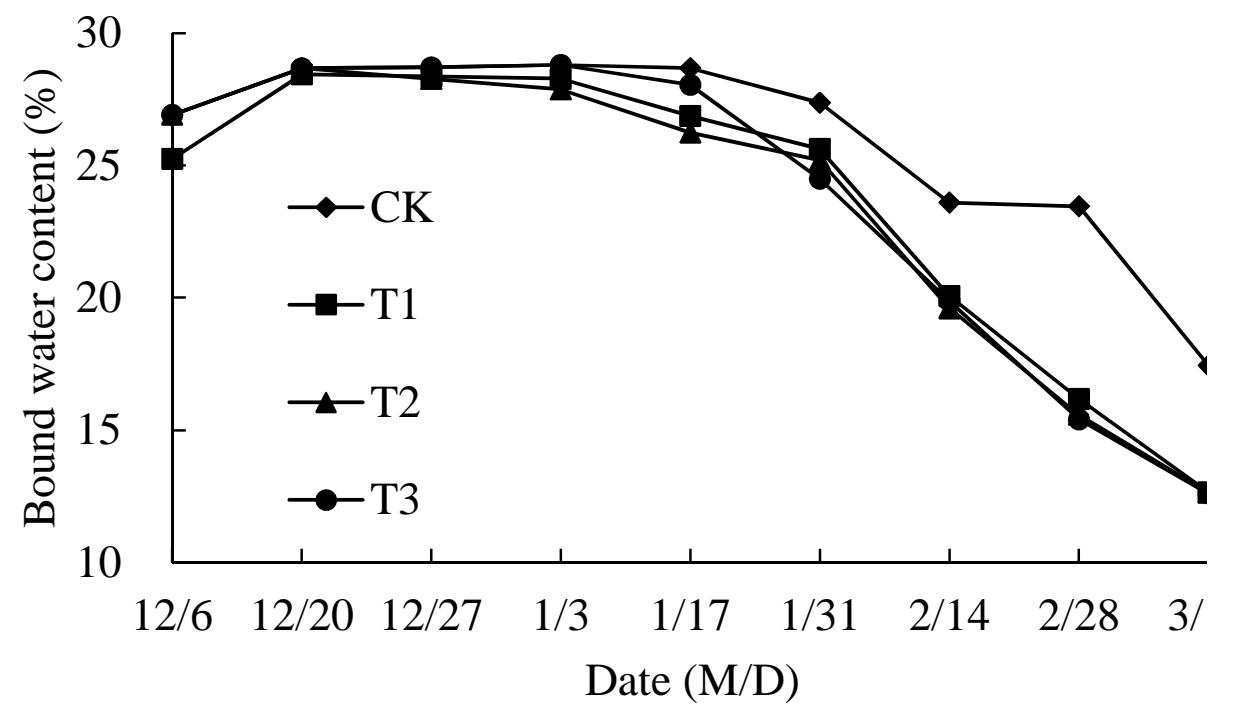

Fig. 3 Change of bound water content

Bound Water/Free Water. The trend of bound water/free water in branches rose first and then decreased from December 6 th to March 13 th (Fig. 4), suggested the same trend as bound water content and the opposite trend of free water and total water content. Cultivation of shed was lower than open field cultivation.

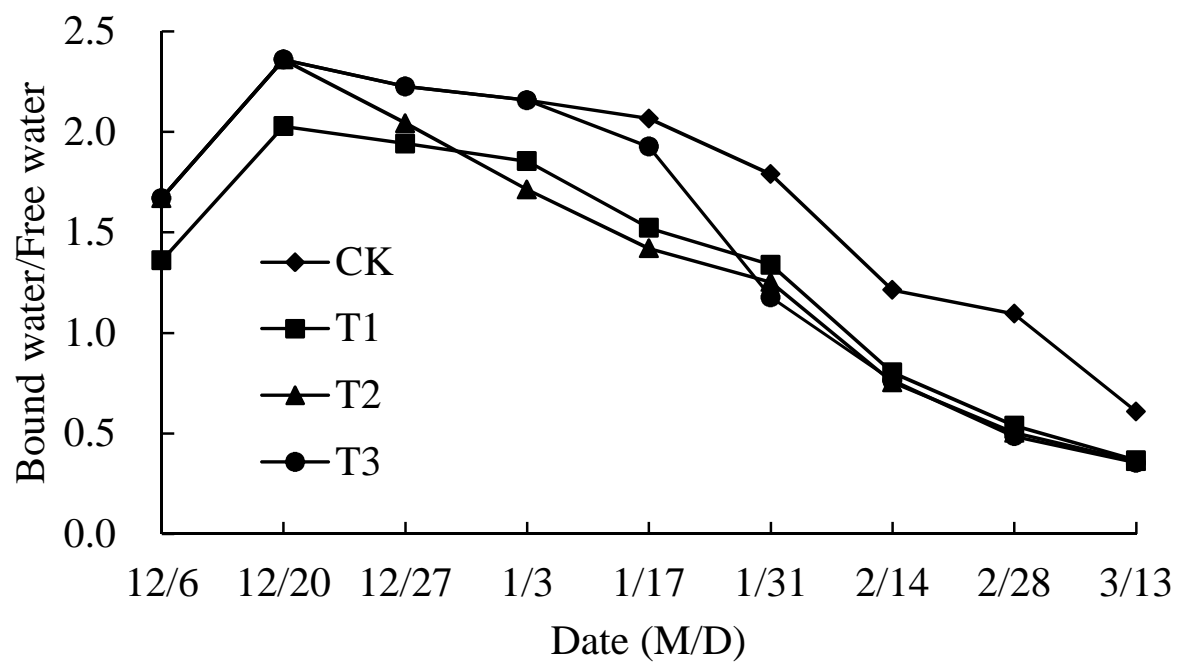

Fig. 4 Change of bound water/free water

Carbohydrate Content. Covering does not change the basic trend of soluble sugar and starch in the branches of Ziziphus jujuba 'Zhanshanmizao', which the content of soluble sugar increased firstly, then decreased, and increased finally (Fig. 5, Fig. 6). The change trend is consistent with the research result of Gao [9]. In all, starch and soluble sugar content showed an opposite trend. Starch can be converted to soluble sugars, so the content was reduced basically or low after the beginning of dormancy. The content of starch of T1, T2, and T3 showed significant decline on January 17 th, while CK on February 14 th, the decrease of shed cultivation was larger than open field cultivation. The 
transformation of starch and soluble sugars may be a sign of the release of the dormant in jujube trees [10]. The change of soluble sugar content was low in natural dormancy, but increased rapidly after natural dormancy released. It cannot be ignored that the change of soluble sugar of shed was greater than field, more important is that the variation time was significantly earlier than field cultivation, the change range of $\mathrm{T} 3$ was biggest.

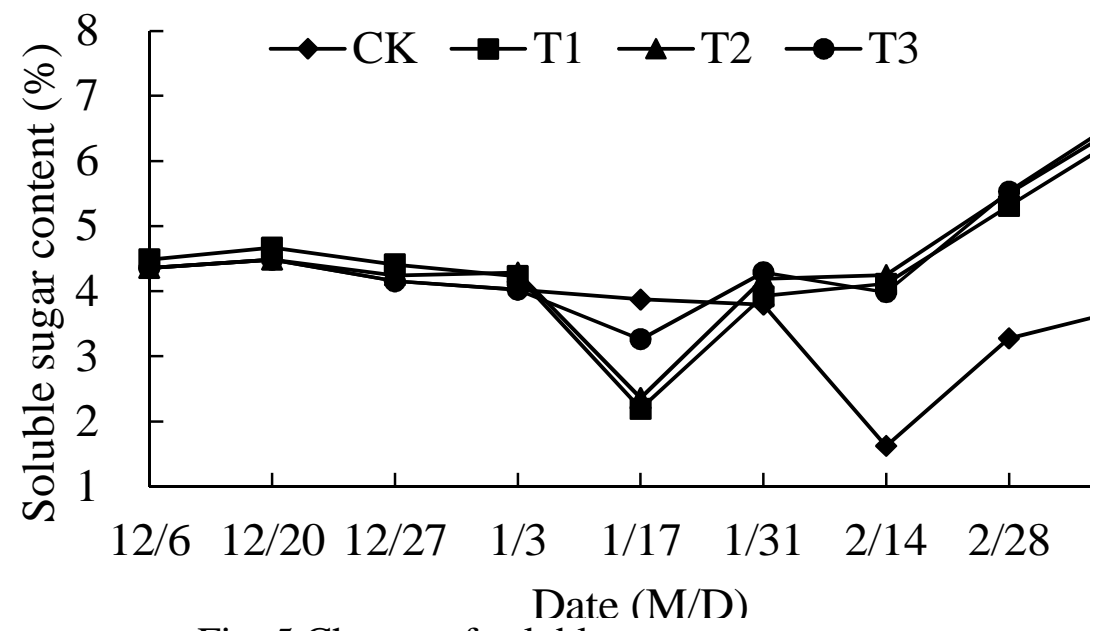

Fig. 5 Change of soluble sugar content

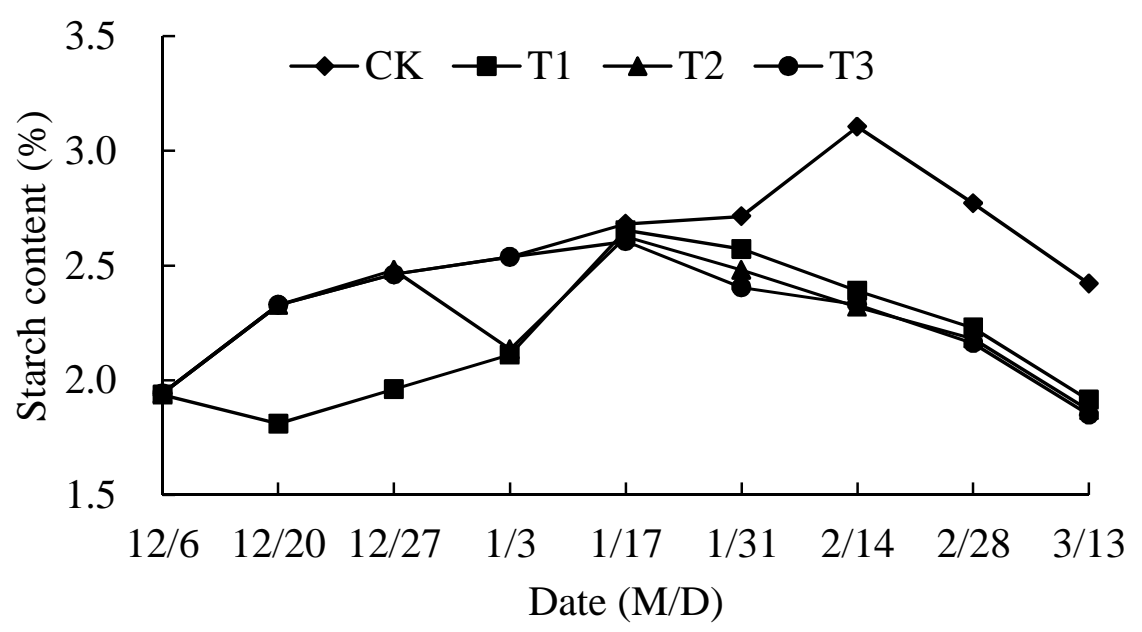

Fig. 6 Change of starch content

Soluble Protein Content and Free Amino Acid Content. The content of soluble protein was lowest at the end of natural dormancy, then increased with the decrease of dormancy and decreased before the germination (Fig. 7). Covering did not change the change trend of soluble protein content or free amino acid content in the process (Fig. 7, Fig. 8). The change trend is consistent with the research consequence of Tian [11], but inconsistent with Li [12]. During natural dormancy, soluble protein content decreased, probably because jujube tree had entered deep dormancy and the low temperature hindered the synthesis of protein or accelerated the hydrolysis of protein. For free amino acid content, T1, T2, and T3 rose slowly after January 17 th, while CK rose slowly after February 14 th. We think this may indicate that covering make the dormancy of jujube released earlier than open field cultivation . 


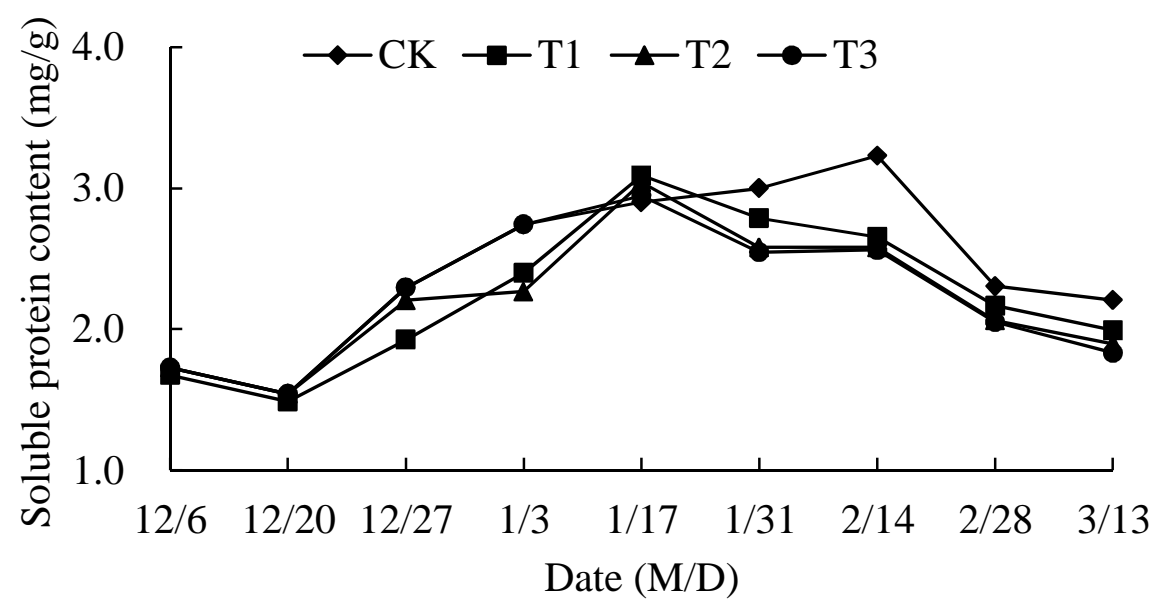

Fig. 7 Change of soluble protein content

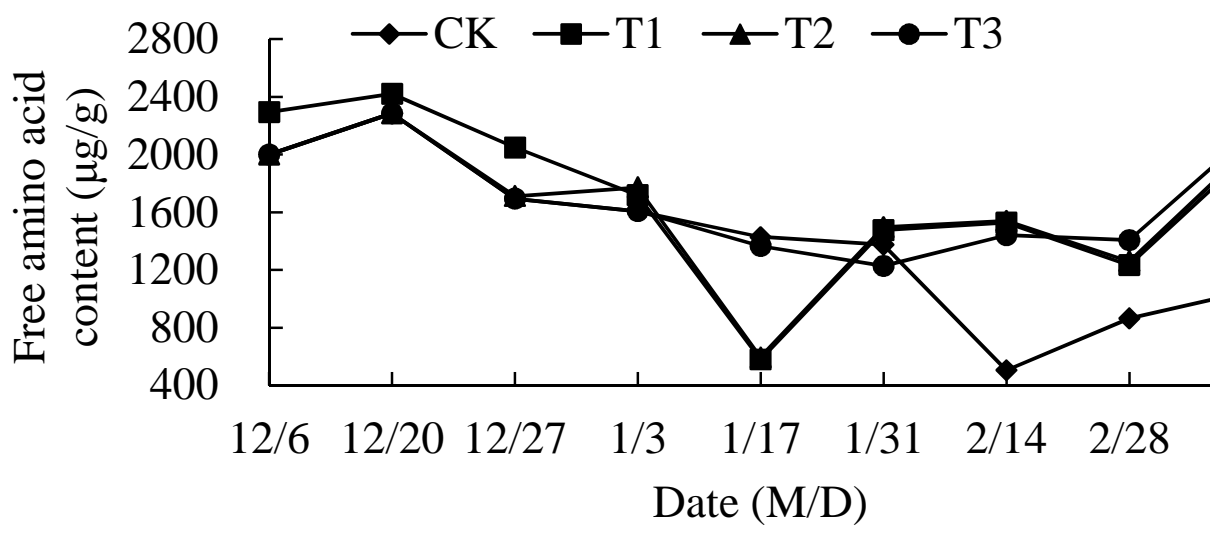

Fig. 8 Change of free amino acid content

\section{Conclusions}

In the course of dormancy, with the deepening of the dormancy of Ziziphus jujuba 'Zhanshanmizao', the total amount of water and free water in branches showed a downward trend, while the bound water and bound water/free water showed an upward trend.

The content of soluble sugar gradually increased during the natural dormant period, peaked when natural dormancy finished, then decreased after entering the forced dormancy, and increased before germination. Starch content showed the opposite trend with soluble sugar content.

The protein content gradually decreased during the dormant period, peaked when the natural dormancy completed, then increased after entering the forced dormancy, and decreased before germination.

As for shed time, covering could significantly change the normal physiology and metabolism of jujube. Covering made Ziziphus jujuba 'Zhanshanmizao' bud germination earlier. Different time comes different result. Covered in January 16 th, 2017 (T3) > covered in December 25 th, 2016 (T2), > covered in December 5 th, 2016 (T1). This may due to the accumulation of chilling requirement on January 16th and forced dormancy comes early, so the effect of T3 is best. The dormancy process of jujube trees can be regulated. 


\section{Acknowledgements}

This work was financially supported by the double subject construction plan of Sichuan Agricultural University and special action plan project of science and technology rich people and strong county of Sichuan Province "integration and demonstration of key technologies for industrialized development of Zizyphus jujuba 'Zhanshangmizao' of Santai County”.

\section{References}

[1] H.B. Wang, D.S. Gao, X.D. Wang and J. Li: Journal of Fruit Science Vol. 23 (2006), p. 91.

[2] S.R. Xia: Beijing Agriculture Vol. 6 (2007), p. 29.

[3] A.Zhang, W.Y.Zheng, T.Chen, Y.L.Fang,H.Yu: Acta Botanica Boreali-Occidentalia Sinica Vol.10 (2012), p. 2075.

[4] M.A. Trejo-Martínez, A. Orozco, G. Almaguer-Vargas, E. Carvajal-Millán and A.A. Gardea: Thermochim Acta, Vol 48 (2009), p.28.

[5] M. Bonhomme, R. Rageau, A. Lacointe, M. Gendraud : Scientia Horticulturae Vol 105 (2005), p. 223.

[6] D. González-Rossia, C. Reig, V. Dovis, N. Gariglio, M. Agustí: Scientia Horticulturae Vol 118 (2008), p. 275.

[7] W.H. D, Z. Zhang, P.H. Zhao, Y.H. Shi: Journal of Fruit Science Vol. 4 (1997), p. 248.

[8] X.K. Wang: The Principle and Technology of Plant Physiology and Biochemistry Experiment (Higher Education Press, Beijing 2004).

[9] C.Y. Gao, A. Zhang, Y.L. Fang, Z.W. Zhang, Q.H. Jin: Acta Botanica Boreali-Occidentalia Sinica Vol. 29 (2009), p. 1200.

[10] R.P. He, M.P. Zhai and J.J. Du: Journal of Fruit Science Vol. 6 (2006), p. 814.

[11] L.L. Tian, J.B. Fang, L.R. Wang and L. Liu: Journal of Southwest University For Nationalities (Natrual Science Edition) Vol. 23 (2006), p. 121.

[12] J. Li, Y.K. Wang: Journal of Fruit Science Vol. 33 (2007), p. 1340. 